\title{
TRES SENTIDOS DE RELATIVISMO
}

\author{
THREE SENSES OF RELATIVISM \\ David Bordonaba Plou \\ Neftalí Villanueva Fernández
}

\begin{abstract}
RESUMEN
El objetivo de este trabajo es doble. Primero, distinguir tres sentidos en los que puede entenderse el término 'relativismo' en el debate acerca del significado de los predicados de gusto y examinar si las posiciones en el debate — contextualismo, contextualismo no deíctico y teorías contexto-evaluativas- son relativistas en alguno de estos sentidos. Segundo, argumentaremos a favor de usar el término 'relativismo' para las teorías contexto-evaluativas por dos razones: i) solo estas teorías asignan verdad a las afirmaciones de gusto de manera relativa; y ii) solo estas teorías prestan suficiente atención al fenómeno de la retractación.
\end{abstract}

PALABRAS CLAVE: contextualismo deíctico, contextualismo no deíctico, teorías contexto-evaluativas, contexto de uso, contexto de valoración.

\begin{abstract}
The aim of this paper is twofold. First, to distinguish three different senses in which the term 'relativism' can be understood in the debate on the meaning of predicates of personal taste, and to examine in what of these senses the different positions taking part in the debate -indexical contestualism, non-indexical contextualism and assessment-sensitivity - are relativist. Second, we will argue for using the label 'relativism' only for assessment-sensitivity for two reasons: i) only these theories asign truth to assertions about taste in a relativist way; ii) only these theories pay enough attention to retraction.
\end{abstract}

KEYWORDS: indexical contextualism, non-indexical contextualism, assessmentsensitvity, context of use, context of assessment. 


\section{INTRODUCCIÓN}

Es sorprendente que uno de los primeros promotores de la educación pública y obligatoria haya pasado a la historia de la filosofía como el responsable de una frase que rara vez oiríamos a día de hoy fuera de un anuncio de viagra: 'El hombre es la medida de todas las cosas'. La frase de Protágoras se asocia con la defensa de algún tipo de subjetivismo, de acuerdo con el cual evalúo las cosas como lo hago simplemente porque así es como me parecen a mí y no debo mayor explicación a nadie, pero el auge del relativismo contemporáneo está asociado con la educación, con la toma en consideración de puntos de vista distintos a los que ahora tengo, ya sean los de otros o los míos propios en algún momento distinto de aquel en el que me encuentro. Solo a través de la experiencia con gente que piensa de manera diferente, o cuando somos capaces de reflexionar acerca de cómo nuestras apreciaciones cambian a lo largo del tiempo, nos volvemos proclives a considerar que tal vez las razones que ahora nos mueven a evaluar un suceso de tal o cual manera puedan verse superadas en el futuro. Y queremos ser esa persona del futuro, esa persona que tiene mejores razones, aunque ello suponga el abandono de nuestras opiniones pasadas, por ejemplo las que ahora sostenemos. El relativismo no es la consagración de la idea de que 'todo vale', sino el resultado de reconocer que el progreso en cuestiones evaluativas es posible.

Contra la "dictadura del relativismo" advertía Benedicto XVI en su primera homilía, del 18 de abril de 2005, en lo que constituye un asalto más del combate que permanentemente se mantiene desde determinados cuarteles filosóficos o amateurs contra el relativismo, seguramente una de las doctrinas filosóficas más vilipendiadas de la historia. Inesperada es por tanto la situación que encontramos en determinados debates recientes, en los que parece que la etiqueta 'relativista' es algo que todas las partes reclaman para sí mismas. Puede pensarse que esto ocurre porque todos los intervenientes en el debate reconocen una caracterización general del relativismo que comparten, lo que podríamos expresar, en términos de Stalnaker de la siguiente manera:

La idea central detrás del relativismo acerca de cualquier concepto concreto o familia de conceptos parece ser que lo que parecería en la superficie ser una afirmación absoluta (la adscripción de un predicado monádico) es en realidad una afirmación relacional (Stalnaker 191, nuestra traducción).

En el debate que analizaremos, la disputa acerca del significado de los predicados de gusto, la mayor parte de las posiciones que encontramos cumplen con 
esta caracterización de Stalnaker y, por tanto, sus defensores pueden con justicia reclamar la etiqueta de 'relativismo' para sus ideas, en este sentido.

Podría ocurrir también, sin embargo, que la reivindicación de la etiqueta 'relativismo' no se corresponda con la mera descripción de un rasgo formal de estas teorías, sino que muestre la intención de estos autores de que sus teorías hagan justicia a las intuiciones relativistas recogidas más arriba, a la idea de que hay perspectivas distintas y de que debemos aspirar a educar nuestro gusto culinario, nuestra apreciación del arte y de la belleza, nuestras disposiciones morales, etc. Esta segunda posibilidad es la que exploraremos en este trabajo y por ello nos centraremos en la noción de retractación: en la posibilidad de evaluar lo que se dijo en un contexto completamente distinto de manera negativa y de exigir a otros que retiren sus evaluaciones como resultado de una discusión fructífera. No todas las posiciones que analizaremos podrán acomodar este requisito de la misma manera

La disputa en la que centraremos nuestro análisis de los posibles usos del término 'relativismo' es el debate sobre predicados de gusto personal (Lasersohn 644). Algunos autores (Kölbel 2003, p. 53; López de Sa 2015, p. 161) usaron el término 'relativismo' para caracterizar a sus teorías porque consideraban que una proposición que incluyera predicados de gusto no era verdadera o falsa simpliciter, sino solo con relación a unas determinadas circunstancias de evaluación. Sin embargo, otros autores (MacFarlane 2007, p. 25; Field 2009, p. 255) objetaron a estos primeros que el término debía reservarse para aquellas teorías que postulaban un nuevo tipo de contexto-dependencia. Según estos últimos, la característica determinante para considerar a una teoría 'relativista' no es considerar que una proposición dada es verdadera o falsa en relación con unas circunstancias desde las cuales se evalúa, sino reconocer que dichas circunstancias pueden depender de un tipo de contexto distinto del contexto de uso: el contexto de valoración. ${ }^{1}$

El objetivo de este trabajo es doble. Primero, distinguiremos varios sentidos en los que puede entenderse el término 'relativismo' en el debate actual respecto al significado de los predicados de gusto. Estos tres sentidos serán: relativismo respecto al contenido $(\mathrm{RCo})$, relativismo respecto a la verdad $(\mathrm{RV})$ y relativismo respecto al contexto

\footnotetext{
1 La diferencia entre contexto de uso y contexto de valoración no debería entenderse como una diferencia entre dos tipos de contexto sino más bien como una diferencia entre dos funciones que un contexto puede desempeñar (MacFarlane 2014, p. 61). En este trabajo nos centraremos en aquellas teorías que señalan una diferencia entre el contexto de uso o contexto de proferencia y el contexto de valoración. Para más información respecto a otras propuestas de bifurcación del contexto (ver Predelli 403; Schlenker 279).
} 
$(R C t x)$. Expondremos las tres familias de teorías que suelen identificarse en la disputa acerca del análisis de los predicados de gusto — contextualimo deíctico, contextualismo no deíctico y teorías contexto-evaluativas- y examimaremos en qué sentido puede decirse de cada una de ellas que es relativista. Dentro de este primer objetivo, se defenderán tres ideas: i) que solo las teorías contextualistas deícticas son relativistas bajo el primer sentido; ii) que bajo el segundo sentido tanto el contextualismo no deíctico como las teorías contexto-evaluativas son teorías relativistas y iii) que bajo el tercer y último sentido solo de estas últimas puede decirse que sean relativistas. Asimismo, clasificaremos las distintas teorías atendiendo a las tres familias antes citadas en un intento de sistematizar la amplia diversidad de propuestas (ver Tabla 1) y de aclarar una cierta confusión en el debate respecto al significado de los predicados de gusto.

En segundo lugar, argumentaremos a favor de reservar el término 'relativismo’ para las teorías contexto-evaluativas. Las teorías contextualistas no deícticas se autodefinen como relativistas porque consideran que el valor de verdad de las proposiciones expresadas por las proferencias que incluyen predicados de gusto solo puede determinarse con relación a unas circunstancias de evaluación. El teórico contexto-evaluativo objeta al contextualista no deíctico que la asignación de condiciones de verdad se lleva a cabo de manera no relativista, ya que se hace en cierto sentido de manera absoluta. Para el contextualista no deíctico, si Alberto dice en $\mathrm{c}_{1}$ 'La lasaña congelada es deliciosa', el parámetro relevante para determinar el valor de verdad de la proposición expresada será el estándar de gusto de Alberto en $\mathrm{c}_{1}{ }^{2}$ al que llamaremos $\mathrm{g}_{1}$. Si, por alguna u otra razón, el gusto de Alberto cambia y en $c_{2}$ dice 'La lasaña congelada es asquerosa', el parámetro relevante para determinar el valor de verdad de la proposición expresada por la proferencia de Alberto en $\mathrm{c}_{1}$, su declaración previa a favor de la lasaña congelada, seguirá siendo $\mathrm{g}_{1}$. Para el contextualista no deíctico 'La lasaña congelada es deliciosa' es verdadera en $\mathrm{c}_{1}$ respecto a $\mathrm{g}_{1}$, y 'La lasaña congelada es asquerosa' es verdadera en $\mathrm{c}_{2}$ respecto a $\mathrm{g}_{2}$. El teórico contexto-evaluativo exige, por su parte, que el parámetro relevante para determinar el valor de verdad de la proferencia hecha en $\mathrm{c}_{1}$ sea el estándar de gusto de Alberto en $\mathrm{c}_{2}$. Cuando uno dice que algo es asqueroso, no puede seguir pensando que es verdad que eso mismo es delicioso. Parece plausible pensar que

2 Este estándar de gusto puede ser el del hablante, aunque también puede ser el de otra persona o incluso puede ser el de algún grupo relevante en el contexto. 
ambas proferencias no pueden ser verdaderas ${ }^{3}$ una vez que el gusto de Alberto ha cambiado, ya que ambas han sido proferidas por el mismo hablante y sus contenidos son incompatibles. Para el teórico contexto-evaluativo, $g_{2}$, el nuevo estándar de gusto de Alberto, no solo es el parámetro relevante para determinar el valor de verdad de su proferencia en $\mathrm{c}_{2}$, sino también el de su proferencia en $\mathrm{c}_{1}$. El argumento crucial en esta disputa tiene que ver con la posibilidad de retirar algo que uno ha dicho previamente: alguien que oyera ambas proferencias podría exigir que Alberto se retractara (MacFarlane 2014, pp. 108-110) de su proferencia en $\mathrm{c}_{1}$. De hecho, cualquier persona que tenga algo de sentido del gusto y un mínimo amor por la gastronomía habría exigido a Alberto que se retractara de su afirmación en $c_{1}$. Además, es posible que Alberto, en vez de decir 'La lasaña congelada es asquerosa', hubiera dicho 'Retiro lo dicho, la lasaña congelada es asquerosa', con lo cual nadie habría tenido derecho a objetarle o exigirle nada.

Retractarse de algo implica asumir que tu estándar de gusto actual es el estándar relevante para determinar el valor de verdad de juicios de gusto hechos en contextos anteriores. Es cierto que los hablantes pueden retractarse por razones que nada tengan que ver con la verdad de las proposiciones expresadas por sus proferencias. Por ejemplo, alguien puede retractarse de una afirmación por razones prudenciales, éticas o por razones prácticas, sin considerar al hacerlo que su afirmación anterior fuera falsa. Aunque sería interesante analizar las características de estos tipos de retractaciones, en este trabajo nos centraremos en aquellas ocasiones en las que los hablantes se retractan de algún juicio de gusto por un cambio en su estándar. Como hemos dicho, retractarse de un juicio de gusto implica considerar que la verdad de las proposiciones que incluyan predicados de gusto no depende del estándar de gusto de quien afirma sino del estándar de gusto de quien evalúa. Esto no supone dejar la verdad de nuestras evaluaciones en manos de quien las recibe, sino reconocer que hay una diferencia entre decir que algo está rico de acuerdo con los gustos de alguien y decir que algo está rico, a secas. Cuando evalúo, al posicionarme con respecto a la evaluación de otro, no puedo saltar por encima de mis estándares. La educación del gusto es solo posible porque admitimos la posibilidad de que lo que ahora nos parece de una manera nos pueda

\footnotetext{
3 MacFarlane más bien habla de que las dos proferencias no pueden ser precisas de manera conjunta. MacFarlane define la precisión (el término que usa MacFarlane es accuracy) de la siguiente manera: 'una actitud o acto de habla que ocurre en $\mathrm{c}_{1}$ es preciso, evaluado desde un contexto $\mathrm{c}_{2}$, solo si su contenido es verdadero al expresarse o proferirse en $\mathrm{c}_{1}$ y evaluarse desde $c_{2}^{\prime}$ (MacFarlane 2014, p. 127, nuestra traducción).
} 
parecer de otra en el futuro, cuando una versión mejor de nosotros, educada, rechace que lo que ahora encontramos delicioso, exquisito, repugnante, insulso, etc. sea tal. Una teoría sobre predicados de gusto que merezca el sobrenombre de relativista debe, al menos, admitir la posibilidad de retractarse, y para esto tiene que permitir que el valor de verdad de algunas proposiciones dependa del estándar de gusto de quien valore. Este es el argumento que desarrollaremos en este trabajo.

El plan para el resto del trabajo es el siguiente: en $\$ 2$ presentaremos los tres sentidos de relativismo; en $\$ 3$ presentaremos el contextualismo deíctico y mostraremos que es relativista con respecto al contenido pero no con respecto a la verdad ni con respecto al contexto; en $\$ 4$ haremos lo propio con el contextualismo no deíctico y mostraremos que no es relativista ni con respecto al contenido ni con respecto al contexto, pero sí con respecto a la verdad; en $\$ 5$ presentaremos las teorías contexto-evaluativas y mostraremos que no son relativistas con respecto al contenido, pero sí con respecto a la verdad y con respecto al contexto; en $\$ 6$ defenderemos que una teoría sobre el significado de los predicados de gusto es relativista si es relativista con respecto al contexto y si puede acomodar el fenómeno de la retractación. En $\$ 7$ extraeremos algunas conclusiones.

\section{TRES SENTIDOS DE RELATIVISMO}

Una teoría puede, en primer lugar, ser relativista con respecto al contenido $(\mathrm{RCo})$. Es decir, puede decirse de una teoría que es relativista si admite la posibilidad de que distintas proferencias de una misma oración expresen distintas proposiciones en distintos contextos. Por ejemplo, si Pedro dice 'Está lloviendo' cuando se encuentra en Granada, la proposición expresada por su proferencia será que está lloviendo en Granada. Si Pedro dice 'Está lloviendo' cuando se encuentra en Zaragoza, la proposición expresada por su proferencia será que está lloviendo en Zaragoza. Del mismo modo, 'El sushi de salmón es delicioso' expresará distintas proposiciones en distintos contextos. Si Pedro dice 'El sushi de salmón es delicioso', la proposición expresada por su proferencia será que el sushi de salmón es delicioso para Pedro o para los estándares de un grupo que incluye a Pedro. Si, por otro lado, Manolo dice 'El sushi de salmón es delicioso', la proposición expresada por su proferencia será que el sushi de salmón es delicioso para Manolo o para los estándares de un grupo que incluye a Manolo.

En segundo lugar, una teoría puede ser relativista respecto a la verdad (RV). Es decir, una teoría puede defender que las proposiciones no son verdaderas o falsas simpliciter, sino solo en relación con unas circunstancias de evaluación determi- 
nadas. El valor de verdad de la proposición expresada por una proferencia de 'El sushi de salmón es delicioso' dependerá del estándar de gusto desde el que se evalúe. Si tomamos el estándar de gusto de Laila o de un grupo que incluye a Laila, la proposición será verdadera. Si tomamos el estándar de gusto de Alberto o de un grupo que incluye a Alberto la proposición será falsa. La distinción entre carácter y contenido (Kaplan 500) ha llegado a ser hoy en día la forma más aceptada de definir este tipo de relativismo. En la teoría kaplaniana solo se requieren dos parámetros en las circunstancias de evaluación, un mundo (W) y un tiempo (T). En la última década, a los parámetros clásicos de las circunstancias de evaluación — mundo, tiempo y lugar — se han sumado otros: estándar de gusto o perspectiva de experimentador para los predicados de gusto (Kölbel 2003, pp. 62-63; Lasersohn 644) y estándar epistémico (MacFarlane 2014, p. 189) para las atribuciones de conocimiento son algunos ejemplos.

Tercero, una teoría puede ser relativista respecto al contexto ( $R C t x)$. Es decir, una teoría puede ser relativista si defiende que los parámetros relevantes para determinar el valor de verdad de una proposición dada no dependen del contexto de uso, sino de otro contexto. Como ya se ha comentado en la Introducción, en este trabajo nos centraremos en el contexto del evaluador, o 'contexto de valoración', como alternativa al contexto de uso. Los estándares que determinan el valor de verdad de nuestras evaluaciones de gusto no dependen necesariamente del contexto en el que la proferencia se lleva a cabo, sino del contexto en el que la proferencia es evaluada. De nuevo, lo que puede resultar verdadero con respecto a unos estándares, puede resultar falso con respecto a otros.

Una vez hechas estas aclaraciones, en las siguientes secciones consideraremos las distintas teorías que han centrado el debate respecto al significado de los predicados de gusto: contextualismo deíctico, contextualismo no deíctico y teorías contexto-evaluativas. Trataremos de ver en cuáles de los sentidos distinguidos puede decirse de estas teorías que son relativistas.

\section{Contextualismo DeíCTiCo}

La idea básica del contextualismo deíctico es que la contexto-dependencia de los predicados de gusto no es distinta a la contexto-dependencia de expresiones deícticas como 'yo', ‘él' o 'aquí'. Para el contextualista deíctico, un predicado de gusto contiene en su forma lógica un hueco de argumento para una perspectiva o un estándar de gusto. Este estándar se recupera del contexto de la misma forma que se recupera la referencia de los deícticos. Aunque predicados como 'divertido’ 
o 'delicioso' puedan parecer monádicos en su forma gramatical superficial, en realidad son predicados diádicos con huecos de argumento ocupados por un deíctico oculto (ver Ludlow 102) que nos lleva a buscar en el contexto el estándar de gusto relevante. Es decir, '... es divertido' sería más bien '... es divertido de acuerdo con el estándar de $x$ ', donde $x$ es un individuo o grupo que incluye al experimentador.

Stanley (Stanley 2000, p. 412; 2002, pp. 151-153) defiende que los elementos de la proposición que se proporcionan contextualmente, por ejemplo el lugar en 'Está lloviendo', son valores de constituyentes en la forma lógica de las oraciones. El test que propone para demostrar que la forma lógica contiene huecos de argumento que escapan al análisis tradicional de la misma recibe el nombre de Binding Criterion (Stanley 2000 Ibid., 2002 Ibid.): si podemos mostrar que hay operadores oracionales de los que podemos hacer depender el elemento contextual, el lugar en el caso de 'está lloviendo', habremos mostrado que este está articulado sintácticamente. Consideremos los siguientes ejemplos:

(1) Está lloviendo.

(2) Cada vez que Enrique saca el mapa, llueve.

(2) puede interpretarse como:

$\left(2^{*}\right)$ Para cada tiempo $t$ en el cual Enrique saca el mapa, llueve en $t$ en el lugar en el que Enrique saca el mapa en $t$.

De acuerdo con el criterio de Stanley, que el lugar donde llueve pueda depender de cuándo Enrique saque el mapa muestra que había una variable en la forma lógica. Si (1) fuera un predicado cero-ádico y no contuviera un lugar de argumento para un lugar, no podría hacerse caer [en (2)] a la variable del lugar bajo el alcance de otro operador oracional.

Glanzberg (Glanzberg 12-15) defiende un 'contextualismo flexible' (ver Hirvonen 105-107) según el cual los predicados de gusto, al igual que otros adjetivos como 'alto' o 'rico', son adjetivos de grado. Un adjetivo como 'alto' es una función

\footnotetext{
4 Stanley no habla en ningún momento de predicados de gusto ni de la posibilidad de que las perspectivas de experimentador o los estándares de gusto puedan ser los valores de constituyentes en la forma lógica de las oraciones. Sin embargo, pensamos que la teoría de Stanley se puede aplicar de igual manera a predicados de gusto como ‘delicioso' o ‘divertido'. De hecho, uno de los tests empleados por Schaffer (Schaffer 192) para mostrar la existencia de huecos de argumento para perspectivas o estándares de gusto es precisamente el mismo test usado por Stanley.
} 
que toma como argumentos individuos y produce como valores grados dentro de una escala de altura. Decir que un sujeto $x$ es alto es decir que es alto en un grado mayor que un estándar de altura $S$ que es determinado por el contexto. Dependiendo del estándar de la escala que seleccione el contexto, 'Quique es alto' puede significar que Quique es alto para ser bijo de sus padres o puede significar que Quique es alto para jugar en un equipo de baloncesto. Para Glanzberg los predicados de gusto funcionan de manera similar. La única diferencia es que los predicados de gusto, además de una clase de comparación, incluyen un hueco de argumento para una clase de experimentador $E$, la cual puede referir al hablante, al destinatario, a la audiencia o a algún otro grupo relevante en el contexto. De esta forma, si Raúl, en un contexto $c_{1}$, dice 'Las películas de Mel Brooks son divertidas', podrá estar comunicando que el grado en el que las películas de Mel Brooks son divertidas es mayor que un estándar de diversión $S$, el de la audiencia de $c$, o que el grado en el que las películas de Mel Brooks son divertidas es mayor que un estándar de diversión $S$, el de Raúl. La teoría de Glanzberg, al igual que la de Stanley, pertenece a las posiciones contextualistas deícticas porque los predicados de gusto tienen huecos de argumento para la clase de experimentador $E$, que forma parte, por tanto, de la proposición expresada. Consideremos el siguiente pasaje:

Si existe tal parámetro [una clase de experimentador $E$ ] en los predicados de gusto personal, se suscita la cuestión de cómo sería su sintaxis. La posición más fuerte, y la que vería más conveniente, es que la clase de experimentador sea un argumento sintáctico genuino del predicado (Glanzberg 15, nuestra traducción).

Schaffer (Schaffer 191) defiende un 'perspectivismo del significado' donde pone de relieve la misma idea que Stanley y Glanzberg: los predicados de gusto tienen huecos de argumento para una perspectiva o estándar de gusto. Consideremos el siguiente pasaje, como ilustración:

Argumentaré que estos dos predicados de gusto ("bueno" y "divertido" respectivamente) introducen sintácticamente argumentos de experimentador reales, los cuales especifican la perspectiva en cuestión (Schaffer 191, nuestra traducción).

En resumen, para las teorías contextualistas deícticas los predicados de gusto contienen huecos de argumento para perspectivas de experimentador o estándares de gusto. Estas perspectivas o estándares son constituyentes de las proposiciones y, como tales, juegan un papel semántico en la determinación del contenido de las proposiciones que incluyan entre sus elementos predicado de gusto. Las proposiciones que expresamos mediante la proferencia de oraciones que contienen predicados de gusto personal cambian de manera sistemática en atención al contexto en el que 
se profieren. $\mathrm{Al}$ igual que el individuo con el que 'ella' contribuye a la proposición depende del contexto en el que la oración en cuestión se profiera, la proposición que exprese una proferencia que incluya 'está rico' como uno de sus componentes dependerá sistemáticamente del contexto en el que está oración se profiera. Precisamente por esta característica las teorías contextualistas deícticas son relativistas respecto al contenido. No serán relativistas respecto a la verdad porque las proposiciones que contengan predicados de gusto serán verdaderas o falsas simpliciter, contendrán como constituyentes todos los elementos necesarios para poder ser evaluadas como verdaderas o falsas. Como veremos, es característico del resto de posiciones relativistas el que algunos de los elementos sin los cuales la proposición no puede evaluarse pasan a formar parte de las circunstancias de evaluación. Tampoco serán relativistas respecto al contexto, porque solo el contexto de uso es necesario para fijar la referencia de los deícticos, incluyendo el deíctico oculto que recupera el estándar de gusto y, por tanto, no apelan al contexto de evaluación.

\section{Contextualismo nO DeíCTICO}

La idea básica del contextualismo no dé́ctico puede resumirse diciendo que las proposiciones que incluyen predicados de gusto no son verdaderas o falsas simpliciter, sino solo con relación a un estándar de gusto o perspectiva de experimentador. A diferencia del contextualismo deíctico, en el contextualismo no deíctico este estándar o perspectiva no será un constituyente de la proposición expresada sino que será uno de los parámetros en relación con los cuales ha de determinarse el valor de verdad de las proposiciones; será, por tanto, parte de las circunstancias de evaluación. De este modo, las proposiciones que incluyen predicados de gusto serán proposiciones neutrales con respecto a la perspectiva de experimentador. Esto nos permite afirmar, entre otras cosas, que dos personas están de acuerdo, que dicen lo mismo o que piensan lo mismo cuando dicen que algo está rico, independientemente de cuáles sean sus respectivos estándares de gusto.

Kölbel (Kölbel 2015, p. 46, 2015b, p. 53) admite la ortodoxia kaplaniana al definir el carácter como una función de contextos a intensiones, y el contenido como una función de circunstancias de evaluación a extensiones. Así, asigna a las expresiones en un contexto de uso una intensión, que es una función I desde un dominio $\mathrm{E} \times \mathrm{A} \times \mathrm{C} \times \mathrm{P}^{5}$ a un conjunto de extensiones $\mathrm{D}$-individuos y valores

${ }^{5} \mathrm{E}=$ un conjunto de expresiones del lenguaje; $\mathrm{A}=$ un conjunto de funciones de asignación; $\mathrm{C}=$ un conjunto de contextos; $\mathrm{P}=$ un conjunto de circunstancias de evaluación. 
de verdad en el caso de oraciones completas-. Como ejemplo, consideremos las definiciones de las siguientes expresiones:

(Yo) $\forall a \in \mathrm{A}, c \in \mathrm{C}, p \in \mathrm{P}:$

$\mathrm{I}\left({ }^{(' y o}, a, c, p\right)=\{x \mid x \text { es el hablante en } \mathrm{W}(c) \text { en } \mathrm{T}(c)\}^{6}$

(Tú) $\forall a \in \mathrm{A}, c \in \mathrm{C}, p \in \mathrm{P}:$

$\mathrm{I}\left({ }^{\prime}\right.$ 'ú', $\left.a, c, p\right)=\{x \mid x$ es interlocutor en $\mathrm{W}(c)$ en $\mathrm{T}(c)\}$

(Ser un dodo) $\forall a \in \mathrm{A}, c \in \mathrm{C}, p \in \mathrm{P}$ :

$\mathrm{I}($ 'ser un dodo', $a, c, p)=\{x \mid x$ es un dodo en $\mathrm{W}(p)$ en $\mathrm{T}(p)\}$

(Ser un jugador de los Lakers) $\forall a \in \mathrm{A}, c \in \mathrm{C}, p \in \mathrm{P}$ :

$\mathrm{I}($ 'ser un jugador de los Lakers', $a, c, p)=\{x \mid x$ es un jugador de los Lakers en $\mathrm{W}(p)$ en $\mathrm{T}(p)\}$

Como puede observarse, existe una diferencia entre las dos primeras definiciones y las dos últimas. La extensión de expresiones como 'yo' o 'este' varía dependiendo de cuál sea el contexto en el que la oración se profiere, pero no varía dependiendo de cuáles sean las circunstancias de evaluación. Al contrario, la extensión de expresiones como 'ser un dodo' o 'ser un jugador de los Lakers' varía dependiendo de cuáles sean las circunstancias de evaluación, pero no varía dependiendo de cuál sea el contexto. Es decir, la extensión de expresiones deícticas como 'yo' o 'este' variará de contexto de uso a contexto de uso. Por ejemplo, si Pedro en $c_{1}$ y Pablo en $c_{2}$ profieren 'Yo soy el responsable', la extensión del pronombre 'yo' en $\mathrm{c}_{1}$ será Pedro, mientras que en $\mathrm{c}_{2}$ será Pablo. Sin embargo, la extensión de expresiones como 'ser un dodo' o 'ser un jugador de los Lakers' no cambiará con el contexto de uso, sino que variará dependiendo de cuál sea el mundo y el tiempo desde el que se evalúa. Por ejemplo, tomando el mundo actual como componente de las circunstancias de evaluación, el concepto 'ser un dodo' es vacío. Sin embargo, si tomamos el mundo tal como era hace 2000 años, la extensión del concepto no es vacía. Por esta razón, se dice que expresiones como 'yo' o 'este' son expresiones dependientes del contexto, mientras que expresiones como 'ser un dodo' o 'ser un jugador de los Lakers' son expresiones que dependen de las circunstancias de evaluación. Todo lo expuesto hasta ahora se aplica de igual a manera a los predicados de gusto. La única modificación que hay que llevar a

\footnotetext{
${ }^{6} \mathrm{~W}(c)$ y $\mathrm{T}(c)=$ funciones que determinan respectivamente el mundo y el tiempo del contexto de uso.
} 
cabo es incluir entre las circunstancias de evaluación, además de un mundo (W) y un tiempo $(\mathrm{T})$, un estándar de gusto $(\mathrm{G})$ :

(Ser delicioso) $\forall a \in \mathrm{A}, c \in \mathrm{C}, p \in \mathrm{P}$ :

$\mathrm{I}$ ('ser delicioso', $a, c, p)=\{x \mid x$ causa al agente saliente en $c$ una experiencia hedónica en $\mathrm{W}(p)$ en $\mathrm{T}(p)$ de acuerdo a $\mathrm{G}(p)\}^{7}$

A pesar de que Lasersohn (Lasersohn 662-663) ${ }^{8}$ defiende un 'relativismo del valor semántico' (ver Glanzberg 2-3 y 8-10), su alternativa pertenece al grupo de teorías contextualistas no deícticas: la perspectiva o estándar de gusto no es parte del contenido, sino de las circunstancias de evaluación:

Todo lo que tenemos que hacer es asignar a palabras como divertido o rico el mismo contenido con relación a diferentes individuos, pero relativizar contextualmente la asignación de valores de verdad a los contenidos, de tal manera que pueda asignarse al mismo contenido distintos valores de verdad con relación a distintos individuos (Lasersohn 662, nuestra traducción).

El relativismo moderado de Recanati (Recanati 2007, p. 37-51) también puede interpretarse como una variedad de contextualismo no deíctico, puesto que distingue entre el lekton y la proposición austiniana, que incluye a las circunstancias de evaluación. Solo la proposición austiniana es verdadera o falsa simpliciter. El lekton solo es verdadero o falso en relación con los parámetros incluidos en las circunstancias de evaluación. Con respecto a ambos niveles de significado cabe expresar acuerdo y desacuerdo. Algunos fenómenos de interés para el relativista, como los modos psicológicos (Recanati 2007, pp. 143-145) forman parte de las circunstancias de evaluación, lo que hace que podamos considerar esta posición como un tipo de contextualismo no deíctico. En lo concerniente a las proposiciones que contienen predicados de gusto personal, Recanati piensa que los estándares de gusto de mi

${ }^{7} \mathrm{G}(p)=$ función que determina el estándar de gusto $g$ respecto a la circunstancia $p$, es decir, el estándar de gusto relevante para determinar el valor de verdad de una proposición dada.

8 Aunque algunos autores sitúan a Lasersohn dentro de las teorías contexto-evaluativas (ver Marques 363), creemos que es más acertado caracterizar su teoría como un tipo de contextualismo no deíctico por las siguientes razones: i) una de las principales ideas de la propuesta de Lasersohn es extender la semántica de doble índice kaplaniana a estándares de gusto, siendo esta una de las motivaciones básicas que dio origen al tipo de contextualismo explicado en la presente sección; y ii) Lasersohn nunca diferencia entre dos tipos de contexto, c1 y c2, por lo que parece que los parámetros para determinar el valor de verdad de las afirmaciones de gusto siempre los proporciona el contexto de uso. 
comunidad (Recanati 2007, pp. 93, 94) son también parte de los parámetros incluidos en las circunstancias de evaluación; suponen un tipo de enriquecimiento pragmático global que afecta a la proposición completa, no a una parte de ella, y, por tanto, no son parte del lekton. Podría argumentarse que, aunque no formen parte del lekton, la inclusión de los estándares de gusto dentro de una de las proposiciones, la proposición austiniana, con respecto a las cuales cabe estar en acuerdo o en desacuerdo acercaría a Recanati al contextualismo deíctico, pero debemos hacer una salvedad importante: Es característico del tipo de contextualismo defendido por Recanati (ver Recanati 2004, pp. 23-36, 2010, pp. 129-133) el que hay procesos pragmáticos que no están guiados por ningún deíctico oculto en la forma lógica. En este sentido, el relativismo moderado, a pesar de ser proposicionalista, no sería deíctico, en el sentido introducido más arriba.

En resumen, el contextualismo no deíctico, en cualquiera de sus variedades, no otorga a la perspectiva de experimentador ningún rol semántico a la hora de determinar el contenido, sino que dicha perspectiva solo entra en juego a la hora de evaluar el valor de verdad de dicha proposición. Por ello, el contextualismo no deíctico no es relativista respecto al contenido, ya que los contenidos de las proposiciones expresadas por oraciones que incluyen predicados de gusto serán neutrales respecto a la perspectiva de experimentador. Sin embargo, sí será relativista respecto a la verdad ya que las proposiciones serán verdaderas o falsas solo con relación a esas circunstancias de evaluación. Al igual que el contextualismo deíctico, el contextualismo no deíctico presentado en esta sección no es relativista respecto al contexto, dado que será siempre el contexto de uso el que determine qué parámetro correspondiente a la perspectiva del experimentador pasa a formar parte de las circunstancias de evaluación.

\section{TEORÍAS CONTEXTO-EVALUATIVAS}

Según MacFarlane (MacFarlane 2014, pp. 52, 62) lo que le convierte a uno en relativista en cuestiones de gusto no es considerar que las proposiciones son verdaderas con respecto a un punto de evaluación, ${ }^{9}$ sino que el parámetro relevante depende del contexto de valoración y no del contexto de uso. Para un contextualista no deíctico, 'El sushi de salmón es delicioso' será verdadera en $c_{1}, c_{2}\langle\mathrm{w}, \mathrm{t}, \mathrm{g}\rangle$ si y solo si el sushi de salmón es delicioso en $c_{1}, c_{2}\left\langle w_{1}, t_{12} z_{1}\right\rangle$. Por el contrario, para el teórico

\footnotetext{
9 A veces se usa el término 'punto de evaluación' para referirse al par contexto-circunstancias de evaluación (MacFarlane 2014, p. 57, nota 19).
} 
contexto-evaluativo, 'El sushi de salmón es delicioso' será verdadera en $c_{1}, c_{2}\langle\mathrm{w}, \mathrm{t}, \mathrm{g}\rangle$ si y solo si el sushi de salmón es delicioso en $c_{1}, c_{2}\left\langle w_{1}, t_{12} g_{2}\right\rangle$. Es decir, para el contextualista no deíctico, el estándar de gusto relevante para determinar el valor de verdad de la proposición expresada por una proferencia de la oración 'El sushi de salmón es delicioso' será g y dicho estándar lo determinará el contexto de uso. Sin embargo, para el teórico contexto-evaluativo el estándar de gusto relevante para determinar el valor de verdad de la proposición expresada por una proferencia de la oración ‘El sushi de salmón es delicioso' será $\mathrm{g}_{2}$ y dicho estándar lo determinará el contexto de evaluación. Para MacFarlane, definir la verdad en un contexto y un índice como hace el contextualista no deíctico es simplemente un recurso técnico para poder definir de manera sistemática la noción de 'verdad en un contexto de uso'. Por esta razón, aceptar que haya oraciones cuyas condiciones de verdad requieran una semántica de doble índice —índices del contexto e índices de las circunstancias de evaluación - no es suficiente para ser un relativista. Aquello que hace relativista a una teoría sobre el significado de los predicados de gusto es admitir la posibilidad de que algunas expresiones de los lenguajes naturales sean sensibles al contexto de valoración. Como MacFarlane afirma:

Voy a sugerir que lo que hace a uno relativista sobre la verdad es el compromiso con la sensibilidad a la valoración de algunas oraciones o proposiciones (MacFarlane 2014, p. 52, nuestra traducción).

Para MacFarlane, el contexto de valoración es el contexto que determina los índices relevantes para evaluar el valor de verdad de las proposiciones que contienen predicados de gusto. Supongamos que David, un enamorado del sushi, dice 'El sushi de salmón es delicioso'. El estándar de gusto relevante para determinar el valor de verdad de la proposición expresada por David no tendrá que ser necesariamente el propio estándar de gusto de David. Si suponemos que Alberto, a quien le repugna el pescado en todas sus formas, oye la afirmación de David, su estándar de gusto será igualmente relevante para determinar el valor de verdad de la proposición. De esta manera, la proposición expresada será verdadera al ser evaluada desde el contexto de valoración de David, pero falsa cuando es evaluada desde el contexto de valoración de Alberto.

La precisión de las proposiciones que contienen predicados de gusto, la noción de verdad con relación a un parámetro de las circunstancias de evaluación, debe también distinguirse de una noción no relacional de verdad. El relativismo de MacFarlane no está comprometido con la idea de que la misma proposición pueda ser verdadera y falsa al mismo tiempo, donde 'verdadero' no tiene carácter 
relacional. Estrictamente hablando, esta alternativa, con la que a veces se asocia al relativismo, no tendría sentido. Solo es posible defender que la misma proposición puede ser verdadera y falsa cuando la noción de verdad que usamos, la precisión, es relacional, y con 'es verdad' siempre queremos decir que es verdadero con respecto a unos estándares.

Field (Field 250-252) defiende un relativismo expresivista. La idea básica de Field es que juzgar que la creencia de un hablante está justificada o es racional es evaluarla, y que este tipo de evaluaciones no son directamente factuales. Es decir, que las evaluaciones —epistemológicas, morales o estéticas — son afirmaciones que implican un parámetro para una norma de evaluación. Por ejemplo, una proferencia de la oración 'Los países occidentales deberían dejar de intervenir en Oriente Medio' en un contexto apropiado puede constituir una evaluación porque es sensible a una norma moral como $\mathrm{N}_{\text {petróleo }}$ : no es correcto intervenir en otros países para apropiarse de las reservas de petróleo del país. Su valor de verdad no puede ser determinado sin tomar en consideración la norma en cuestión. Una afirmación de 'El origen del mundo de Gustave Courbet es horrible' es una evaluación porque es sensible a una norma estética como $\mathrm{N}_{\text {contenidosexual }}$ las obras de arte con contenido sexual explícito no son bellas. Las evaluaciones epistemológicas, morales o estéticas son expresiones contexto-dependientes, ya que para determinar su valor de verdad es necesario tener en cuenta una norma determinada por el contexto. Pero, al igual que en el caso del relativismo defendido por MacFarlane, el contexto que importa no es el contexto de uso, sino el contexto de valoración:

He puesto de relieve anteriormente que existen dos grandes diferencias entre el tipo de relatividad implicado en afirmaciones sobre la justificación y el tipo de relatividad implicada en oraciones contextuales habituales como 'Está lloviendo'. La primera, la cual he mencionado pero aún no he explicado, es relatividad contextual, frente a la relatividad del evaluador. La segunda, implícita en los últimos párrafos, es que mientras que la relatividad de 'Está lloviendo' no es en absoluto controvertida, la relatividad de las afirmaciones epistemológicas está muy lejos de ello: para los realistas epistemológicos es sin duda cuestionable, y al menos es contraria a las opiniones de la gente corriente. (Field 254, nuestra traducción).

El tipo de contexto-dependencia de expresiones como 'Está lloviendo' es muy distinta al tipo de contexto-dependencia de expresiones como 'Matar está mal' o 'Saturno devorando a un hijo es un cuadro magnífico'. En el primer caso podría decirse que el hablante que profiere 'Está lloviendo' está haciendo una afirmación sobre un lugar específico. Sin embargo, en el caso de afirmaciones evaluativas que son sensibles a una norma, el hablante no está haciendo una afirmación sobre la 
norma específica, sino una afirmación que involucra una norma. Por ejemplo, en el caso de una evaluación estética como 'Saturno devorando a un hijo es un cuadro magnífico’, el hablante está haciendo una afirmación sobre un objeto, el cuadro pintado por Goya y, al mismo tiempo, está expresando su aprobación de dicho cuadro.

Otra diferencia entre la contexto-dependencia de expresiones como 'Está lloviendo' y expresiones como 'Saturno devorando a un hijo es un cuadro magnífico' se muestra en las consecuencias que tiene el hacer explícito el parámetro relevante en cada caso. Si alguien que está en Granada en vez de decir 'Está lloviendo' dijera 'Está lloviendo en Granada', no estaría comunicando nada distinto. Hacer explícito el parámetro no afecta a la proposición expresada. Sin embargo, si en vez de decir 'Los países occidentales deberían dejar de intervenir en Oriente Medio' alguien dijera 'Los países occidentales deberían dejar de intervenir en Oriente Medio de acuerdo a la legislativa de la ONU', la afirmación podría ser una muy distinta. Podría pasarse de estar evaluando lo que los países occidentales deberían hacer, a describir lo que deberían hacer si siguieran determinada norma. Para Field, la relatividad a la norma puede perderse cuando se hace explícita la norma en cuestión: 'una afirmación respecto a lo que está justificado de acuerdo a una norma específica sería una afirmación directamente factual, sin fuerza evaluativa alguna' (Field 251-252, nuestra traducción).

TABLA 1

Posiciones teóricas sobre el significado de los predicados de gusto

\begin{tabular}{|c|c|c|}
\hline $\begin{array}{c}\text { Contextualismo deíctico } \\
\text { (RCo/NRV/NRCtx) }\end{array}$ & $\begin{array}{c}\text { Contextualismo no deíctico } \\
\text { (NRCo/RV/NRCtx) }\end{array}$ & $\begin{array}{c}\text { Tas }^{\text {Contexto-Evaluativas }} \\
\text { (NRCo/RV/RCtx) }\end{array}$ \\
\hline $\begin{array}{c}\text { Binding Criterion } \\
\text { (Stanley 2000, 2002) }\end{array}$ & $\begin{array}{c}\text { Relativismo no deíctico } \\
\text { (Kölbel 2003) }\end{array}$ & $\begin{array}{c}\text { Sensibilidad a la valoración } \\
\text { (MacFarlane 2007, 2014) }\end{array}$ \\
\hline $\begin{array}{c}\text { Contextualismo flexible } \\
\text { (Glanzberg 2007) }\end{array}$ & $\begin{array}{c}\text { Relativismo del valor semántico } \\
\text { (Lasersohn 2005) }\end{array}$ & $\begin{array}{c}\text { Relativismo expresivista } \\
\text { (Field 2009) }\end{array}$ \\
\hline $\begin{array}{c}\text { Perspectivismo del significado } \\
\text { (Schaffer 2011) }\end{array}$ & & \\
\hline
\end{tabular}

En conclusión, para Field, al igual que para MacFarlane, una teoría sobre los predicados de gusto es relativista si los parámetros relevantes para determinar el valor de verdad de las proposiciones que incluyen este tipo de predicados dependen del contexto de valoración y no del contexto de uso. Las teorías contextoevaluativas no serán relativistas respecto al contenido, ya que la perspectiva de experimentador o el estándar de gusto no juegan ningún papel semántico en la de- 
terminación de la proposición expresada. Sí serán relativistas respecto a la verdad, ya que el valor de verdad de las proposiciones que incluyan predicados de gusto se determinará solo en relación con una circunstancia de evaluación. Y también serán relativistas respecto al contexto porque las circunstancias de evaluación dependerán del contexto de valoración y no del contexto de uso.

\section{6. ¿QUÉ IMPLICA SER RELATIVISTA RESPECTO A LOS JUICIOS DE GUSTO?}

Lyons (Lyons 109) distinguió entre un 'relativismo del agente', según el cual las acciones de alguien solo pueden evaluarse con respecto a los estándares de esa persona, y un 'relativismo del evaluador', de acuerdo con el cual son los estándares de quien emite el juicio los que han de jugar un papel en la determinación de la verdad de la evaluación. En ambos casos son variedades de lo que aquí hemos llamado 'relativismo del contenido'. La característica general del relativismo, según Lyons, es 'la aceptación de más de un conjunto de estándares morales básicos' (Lyons Id. 110). A medida que aprendemos que los puntos de vista con respecto a los que la gente juzga pueden variar, porque nos educamos con respecto a otras culturas, a la historia o a la propia diversidad de las comunidades en las que nos incluimos, crece la tendencia a adoptar una posición relativista con respecto a los juicios de otros. Hay acciones que nos es extraordinariamente difícil juzgar de acuerdo con nuestros criterios, porque la sociedad en la que ocurrieron nos resulta demasiado extraña. En este sentido, el relativismo es, como afirma Bernard Williams, una consecuencia natural de la educación (Williams 158 y ss.). Pero educarse acerca de la diversidad de estándares no es suficiente para ser relativista. Admitir que puede haber distintos puntos de vista es solo una condición necesaria del relativismo. Ser relativista, defenderemos en esta sección, requiere que seamos capaces de dar cuenta del tipo de fenómenos que resultan cruciales para enmarcar la actitud de quienes reclaman para sí la etiqueta de 'relativistas'.

El criterio de Lyons, como el de Stalnaker mencionado al principio de este trabajo, es insuficiente. Lo que hace a una teoría ser relativista no es el hecho de que se reconozcan distintos parámetros, o que la verdad se convierta en una noción relacional, que enlace proposiciones con esos parámetros diferentes, sino más bien la manera en la que se da cuenta de ese carácter relacional. Todas las posiciones que hemos analizado en este trabajo reconocen la existencia de diferentes parámetros y contienen nociones relacionales de verdad, pero relativizan de maneras crucialmente distintas. En esta sección argumentaremos a favor de usar el término 
'relativismo' para designar a las teorías contexto-evaluativas. Dos de las razones que aduciremos son las siguientes: i) la manera en que asignan verdad las teorías contextualistas no deícticas, al igual que las deícticas, no es relativa, sino absoluta; ii) las teorías contextualistas no deícticas no dan suficiente importancia al fenómeno de la retractación, un fenómeno que suele ir asociado de manera peculiar al uso de predicados evaluativos, como los de gusto, y que solo tiene sentido si consideramos que el valor de verdad de los juicios de gusto es siempre relativo.

Como ya se comentó en $\$ 1$ y $\$ 5$, una de las razones por las que no puede decirse que el contextualismo no deíctico merezca el sobrenombre de 'teoría relativista' es la manera en que asigna verdad a las proposiciones. Como veíamos, si Alberto en $c_{1}$ dice 'La lasaña congelada es deliciosa', para el contextualista no deíctico el parámetro relevante para determinar el valor de verdad de la proposición expresada por la proferencia de Alberto será $g_{1}$, el estándar de gusto de Alberto en $c_{1}$. Si pasado el tiempo el gusto de Alberto cambia y ya no piensa que la lasaña congelada sea deliciosa, para el contextualista no deíctico el parámetro relevante para determinar el valor de verdad de su proferencia en $c_{1}$ seguirá siendo $g_{1}$. Esto hace que pueda decirse que el contextualista no deíctico asigna valores de verdad a las proposiciones que incluyen predicados de gusto de manera absoluta. Una vez que se ha determinado el valor de verdad de una proposición dada, este permanecerá inalterado. Para que una teoría sea relativista es necesario que reconozca que el valor de verdad de las afirmaciones o juicios de gusto no se determina de una vez por todas, que puede variar dependiendo de quién lo evalúe. ${ }^{10}$ Ya que el parámetro relevante para determinar el valor de verdad de un juicio de gusto será el estándar de gusto determinado por el contexto de valoración y, puesto que pueden existir incontables evaluadores potenciales, el valor de verdad de los juicios de gusto será siempre relativo.

La plausibilidad de la idea defendida por las teorías contexto-evaluativas de que el valor de verdad de los juicios de gusto tiene un carácter relativo se ve reflejado en la retractación, uno de los fenómenos que suele ir asociado con el uso de predicados de gusto. Una de las formulaciones más claras del fenómeno de la retractación se debe a MacFarlane:

Por 'retractación' me refiero al acto de habla que uno lleva a cabo al decir 'Retiro eso' o 'Me retracto de eso'. El objetivo de una retractación es otro acto de habla que puede ser una aseveración, una pregunta, una orden, una oferta, o un acto de

${ }^{10}$ El valor de verdad de los juicios de gusto incluso puede variar si es la misma persona la que lo evalúa si, por ejemplo, suponemos que el gusto de dicha persona cambia con el paso del tiempo. 
habla de otro tipo. El efecto de retractarse es "deshacer" los cambios normativos producidos por el acto de habla original (MacFarlane 2014, p. 108, nuestra traducción).

El fenómeno de la retractación nos permite examinar si una teoría sobre los predicados de gusto es relativista o no. Para el contextualismo no deíctico dar una explicación de la retractación no es ni siquiera una opción. Si el parámetro relevante para determinar el valor de verdad en cada caso es el estándar de gusto del hablante en el momento de la proferencia, ¿por qué debería cualquier hablante retractarse de una afirmación de gusto sincera si sus gustos cambian? Es decir, si Alberto en $c_{1}$ dice 'La lasaña congelada es deliciosa', el estándar de gusto para determinar el valor de verdad de la proposición expresada por su proferencia será el estándar de gusto de Alberto en $c_{1}$, es decir, $g_{1}$. Si, pasado el tiempo, Alberto dice en $c_{2}$ 'La lasaña congelada es asquerosa', el estándar de gusto para determinar el valor de verdad de la proposición expresada por su proferencia será el estándar de gusto de Alberto en $c_{2}$, es decir, $g_{2}$. Pero, ¿por qué debería Alberto evaluar "de manera retrospectiva" su afirmación? Es decir, ¿por qué debería evaluar la afirmación hecha en $c_{1}$ con el estándar de gusto que tiene en $c_{2}$ ? Nada en las teorías contextualistas no deícticas exige, ni siquiera permite, que un hablante evalúe una afirmación hecha en un contexto $c_{1}$ usando una circunstancia de evaluación de un contexto $c_{2}$. El único estándar de gusto relevante para determinar el valor de verdad de una afirmación de gusto será el estándar de gusto del hablante en el contexto en el que profiera dicha afirmación.

Sin embargo, para el relativista la retractación es obligatoria. Como ya hemos dicho, para el relativista el estándar de gusto relevante para determinar el valor de verdad de una proposición o juicio de gusto vendrá fijado por el contexto de valoración. Si nuestro gusto cambia con el paso del tiempo, nuestro contexto de valoración también cambiará, y el nuevo contexto de valoración será el que determine el valor de verdad de nuestros juicios de gusto, tanto los actuales como los pasados. Si, por ejemplo, el gusto de Alberto cambia, y pasa de pensar que la lasaña congelada es deliciosa a pensar que es asquerosa, entonces, defiende el relativista, Alberto debería retractarse de su afirmación anterior en la que manifestaba su predilección por la lasaña congelada. Sin embargo, podemos preguntarnos si existen razones para otorgar tanta importancia al fenómeno de la retractación, como hace el relativista. Por ejemplo, cuando a veces a algunas personas se les pide que se retracten, se defienden diciendo que la demanda que se les hace es injusta porque el gusto que tenían en aquel momento era el que era. Ahora simplemente tienen un gusto distinto, y no ven razones para retractarse de su afirmación anterior. Es cierto que esta es 
una estrategia habitual usada por muchas personas, pero también es cierto que es una estrategia de "salvaguarda". Si uno no está dispuesto a retractarse de afirmaciones anteriores cuando su gusto cambia, entonces tampoco tiene derecho a aspirar a ningún tipo de avance o mejora en cuestiones de gusto. Del mismo modo, tampoco tendrá derecho a reclamar para sí una mejor consideración por parte del resto de la sociedad en cuestiones de gusto; por ejemplo, a ser tomado como referente en un debate abierto sobre tales cuestiones. Si uno no arriesga nada en la arena pública, si nuestras afirmaciones no son susceptibles de ser revisadas y reevaluadas, entonces tampoco tendremos derecho a ser honrados con los laureles de la victoria. El progreso en cuestiones de gusto está íntimamente relacionado con la posibilidad de que los hablantes se retracten de afirmaciones anteriores y, por ello, con la idea de que el valor de verdad de las afirmaciones o juicios de gusto no se determina de una vez por todas, sino que puede variar dependiendo de quién lo evalúe.

\section{CONCLUSIONES}

Hay dos tipos de crímenes "perfectos" y a ellos corresponden clases distintas de villanos. Están, por una parte, los crímenes que sabemos que ocurrieron. En ocasiones los responsables de estos son conocidos, pero desaparecen de la faz de la tierra y parecen imposibles de encontrar, como John List, que llevó a cabo un elaborado plan de escape tras asesinar a su mujer y a sus hijos y consiguió pasar desapercibido durante 18 años, viviendo bajo una identidad falsa. Otras veces, quien lleva a cabo el crimen consigue ocultar su identidad no solo después de la comisión del mismo, sino incluso durante el desarrollo del mismo, dejando a sus perseguidores con poco más que un alias. Así ocurrió con 'D. B. Cooper', nombre otorgado al secuestrador de un vuelo en 1971, que desapareció con el equivalente a un millón de euros actuales de rescate, saltando en paracaídas en pleno vuelo, y de quien nunca se volvió a saber. Conocida o no su identidad, estos criminales consiguen evadir a la justicia después de haber llevado a cabo crímenes cuya existencia no puede ponerse en duda: la familia de John List fue asesinada y el Boeing 727 de 'D. B. Cooper' fue efectivamente secuestrado. Sin embargo hay, por otra parte, crímenes que no solo resultan "perfectos" porque sus perpetradores han conseguido de manera efectiva esquivar la persecución, sino que lo son también porque la mera existencia del hecho es imposible de certificar. La reputación terrible que acompaña a Keyser Söze en la película Sospechosos habituales no solo proviene del secretismo con el que se rodea a su figura, sino también del carácter no verificable de los hechos que se le atribuyen. Keyser Söze es el criminal "perfecto" 
porque sirve un propósito concreto, la intimidación dentro de una estructura criminal, sin estar sujeto a ninguna de las consecuencias asociadas con la comisión de un crimen: su crimen y su figura no son en realidad más que una ficción. Keyser Söze no solo no ha pagado jamás por su crimen, sino que está necesariamente libre de las consecuencias de este. Con el relativismo ocurre que uno empieza pensando que es John List, el asesino de su familia, luego piensa que es D. B. Cooper, una mera etiqueta para el perpetrador de un secuestro, y acaba convencido de que no es más que Keyser Söze, una ficción antagónica creada con el solo objetivo de hacer que las ideas propias aparezcan con el marchamo de lo moralmente heroico.

El relativismo como escurridizo autor material conocido de un crimen que sabemos que existió, John List, es el que se corresponde con el supuesto relativismo del agente descrito por Lyons (Lyons 109), el derivado del avance de la antropología, que identificamos con la idea de que las nociones normativas solo pueden ser usadas para expresar proposiciones que contienen los estándares de quienes llevan a cabo la acción. Existe de hecho quien piensa de esa manera, pero su uso del término 'relativismo' no se corresponde con el que reivindican los autores que hemos analizado en este trabajo ni tampoco coincide plenamente con el sentido que utilizan quienes ven en el relativismo una peligrosa ideología que es necesario combatir. 'Relativismo' como etiqueta para un criminal que no conseguimos identificar correctamente, como ocurre con D. B. Cooper y con la caracterización general de Stalnaker, es poco útil como herramienta heurística, puesto que es compatible con demasiadas posiciones teóricas y, de nuevo, no satisfaría ni a los defensores de la posición ni a sus detractores. El relativismo que juega el papel de 'hombre del saco' en el imaginario de distintos tipos de fundamentalismo, esencialmente de origen religioso, es Keyser Söze, alguien que no existe, que ha llevado a cabo crímenes que nunca ocurrieron. El relativista a quien deberían oponerse, sin embargo, no es Keyser Söze, sino alguien igualmente peligroso para sus intereses: Protágoras, el defensor de la educación y del progreso, el que cree que nuestras teorías acerca de lo rico, lo bello y lo bueno deben acomodar la posibilidad de que nos convirtamos en mejores personas.

\author{
David Bordonaba Plou \\ Universidad de Granada \\ davidbordonaba@gmail.com \\ Neftali Villanueva Fernández \\ Universidad de Granada \\ anefcor@gmail.com
}




\section{BIBLIOGRAFÍA}

FiELD, H. (2009): "Epistemology without metaphysics", Philosophical Studies, nº 143(2), pp. 249-290.

GlanzBerg, M. (2007): “Context, content, and relativism”, Philosophical Studies, n’ 136(1), pp. 1-29.

Hirvonen, S. (2014): Predicates of personal taste and perspective dependence. PhD thesis, UCL.

KAPLAN, D. (1989): “Demonstratives", en J. Almog, J. Perry \& H. Wettstein (eds), Themes from Kaplan, Oxford: Oxford University Press, pp. 481-563.

KÖlbel, M. (2003): "Faultless disagreement", Proceedings of the Aristotelian Society, no 104, pp. 53-73.

KÖLBEL, M. (2009): “The evidence for relativism”, Synthese, no 166(2), pp. 375-395.

KÖLBEL, M. (2015): "Relativism 1: Representational content", Philosopby Compass, no 10(1), pp. 38-51.

KÖlBEL, M. (2015b): "Relativism 2: Semantic content”, Pbilosopby Compass, no 10(1), pp. 52-67.

LASERSOHN, N. (2005): "Context dependence, disagreement and predicates of personal taste", Linguistics and Philosophy, $\mathrm{n}^{\circ}$ 28, pp. 643-686.

LÓPEZ, D. (2015): "Expressing disagreement: a presuppositional indexical contextualist relativist account", Erkenntnis, no 80, pp. 153-165.

LuDLOw, P. (1995): "Logical form and the hidden-indexical theory: A reply to Schiffer", The Journal of Philosophy, $\mathrm{n}^{\circ}$ 2, pp. 102-107.

LYONS, D. (1976): "Ethical Relativism and the Problem of Incoherence", Ethics, n 86(2), pp. 107-121.

MacFarlane, J. (2007): "Relativism and disagreement", Pbilosophical Studies, no 132(1), pp. 17-31.

MacFarlane, J. (2014): Assessment-Sensitivity: Relative Truth and its Applications, Oxford: Oxford University Press.

MARQues, T. (2014): "Relative correctness", Philosophical Studies, no 167, pp. 361-373.

Predelli, S. (1998): "Utterance, interpretation and the logic of indexicals", Mind and Language, $n^{\circ} 13(3)$, pp. 400-414.

Recanati, F. (2004): Literal meaning, New York: Cambridge University Press.

Recanati, F. (2007): Perspectival Thought: A Plea for (Relativism), New York: Oxford University Press.

Recanati, F. (2010): Truth-Conditional Pragmatics, New York: Oxford University Press.

SCHAFFER, J. (2011): "Perspective in taste predicates and epistemic modals", en A. Egan \& B. Weatherson (eds.), Epistemic modality, New York: Oxford University Press, pp. 179-226.

SCHLENKER, P. (2004): "Context of thought and context of utterance: A note on free indirect discourse and the historical present", Mind and Language, $\mathrm{n}^{\circ}$ 19(3), pp. 279-304.

StAlNAKeR, R. (2014): Context, New York: Oxford University Press.

STANLEY, J. (2000): "Context and logical form", Linguistics and philosophy, $\mathrm{n}^{\circ}$ 23, pp. 391-434. StANLEY, J. (2000): "Making it articulated", Mind and Language, $\mathrm{n}^{\circ}$ 17(1 \& 2), pp. 149-168. WiLLIAMs, B. (1985): Ethics and the limits of philosophy. Abingdon: Taylor \& Francis. 